\title{
Closed-Form Formulae for Fractional Gradient Tensor of Magnetic Dipole
}

\author{
Ruijian Wang and Yangyi Sui
}

\begin{abstract}
Fractional calculus can be regarded as an important supplement to integer calculus, and has been gradually applied in physics, engineering and so on. In this paper, we define the fractional magnetic gradient tensor of a magnetic dipole, and derive its analytic expressions by using the rule of the composition of fractional-order and integer-order derivatives. Then we verify the analytic expressions by comparing with the results of the numerical method. When the order $\alpha$ of fractional derivatives approaches zero, the fractional magnetic gradient tensor of a magnetic dipole becomes the matrix composed of three magnetic field components. When $\alpha$ approaches one, the fractional magnetic gradient tensor becomes the standard magnetic dipole tensor. This trend shows that fractional derivatives and integer derivatives are consistent. Magnetic gradient tensors have larger attenuation with higher derivative orders when increasing the distance between the observation point and the magnetic source. Therefore, the limited resolution of the magnetic sensors causes a large blind area in a survey, which can be compensated by measuring the fractional magnetic gradient tensor. In addition, each component of the fractional tensor is independent and has great potential of solving the multiple solution problems of the localization of a magnetic dipole.
\end{abstract}

Index Terms-Fractional calculus, Magnetic dipole, Fractional magnetic gradient tensor

\section{INTRODUCTION}

F ractional calculus has a history of more than 300 years. Its derivative orders can be set as a non-integer value, so it is often regarded as the transition between integer-order derivatives. In recent decades, it has been gradually applied in physics, engineering and other fields [1]. Cooper etc. used the numerical method to calculate the fractional derivatives of the measured gravity and magnetic data to obtain the tradeoff between resolution and noise [2]. Engheta proved analytically that fractional-order multipoles of electric-charge densities and their scalar potential distributions between point monopoles and point dipoles [3].

Magnetic dipole is also a widely used basic model. There are analytic formulae for integer order gradient tensors of the magnetic dipole [4], while no analytic fractional expressions. Therefore, we aim to generalize the integer order magnetic gradient tensor of the magnetic dipole to that of fractional. We

This work was supported by the National Natural Science Foundation of China under Grant No. 42074217.

R. Wang and Y. Sui are with the Key Laboratory of Geo-exploration define the fractional magnetic gradient tensor and then derive its analytic expressions, which is helpful to explore new measurement and inversion methods. For example, the magnetic gradient tensor is inversely proportional to $r^{4}(r$ is the distance between the observation point and the magnetic source), while the magnetic field is inversely proportional to $r^{3}$ [4]. The attenuation of the magnetic gradient tensor is obviously faster than that of the magnetic field, which causes the available tensor measurement area is much smaller than that of the magnetic field with the limited resolution of magnetic sensors. With the help of the fractional magnetic gradient tensor, we not only can extend the integer tensor measurement area, but also obtain the abundant fractional gradient information. Another example is the localization of a magnetic dipole [5], since only five of the nine components of the magnetic gradient tensor are independent, there are multiple solutions in the inversion problems, which can be solved by the additional information provided by the fractional magnetic gradient tensor.

\section{Derivation Of Fractional Magnetic GRADIENT TENSOR OF MAGNETIC DiPOLE}

The definition of the magnetic gradient tensor $\boldsymbol{G}^{(2)}$ of a magnetic dipole is [6]

$$
\boldsymbol{G}^{(2)}=\left[\begin{array}{lll}
\frac{\partial^{2} \varphi^{(0)}}{\partial x \partial x} & \frac{\partial^{2} \varphi^{(0)}}{\partial x \partial y} & \frac{\partial^{2} \varphi^{(0)}}{\partial x \partial z} \\
\frac{\partial^{2} \varphi^{(0)}}{\partial y \partial x} & \frac{\partial^{2} \varphi^{(0)}}{\partial y \partial y} & \frac{\partial^{2} \varphi^{(0)}}{\partial y \partial z} \\
\frac{\partial^{2} \varphi^{(0)}}{\partial z \partial x} & \frac{\partial^{2} \varphi^{(0)}}{\partial z \partial y} & \frac{\partial^{2} \varphi^{(0)}}{\partial z \partial z}
\end{array}\right]=\left[\frac{\partial^{2} \varphi^{(0)}}{\partial x_{i} \partial x_{j}}\right]=\left[\frac{\partial B_{x_{i}}^{(1)}}{\partial x_{j}}\right]
$$

where $\varphi^{(0)}$ is the potential of the magnetic dipole, and the superscript implies the derivative order relative to the potential $\varphi$. $x_{i}$ or $x_{j}$ represents to the symbol $x, y$, and $z$ when $i, j=1,2$, and 3 , and it also indicates the number of row or column of the matrix. $G_{i j}^{(2)}$ is the second partial derivative of the potential, or the derivative of the magnetic field component $B_{x_{i}}^{(1)}$ with respect to $x_{j}$. In a source free region $\nabla \cdot \boldsymbol{B}=0$ and $\nabla \times \boldsymbol{B}=0$, so there are only five independent tensor components.

Let the Riemann-Liouville fractional derivative of $B_{x_{i}}^{(1)}$ with respect to $x_{j}$ be the fractional gradient of the magnetic dipole, which is represented as $B_{x_{i} x_{j}}^{(1+\alpha)}={ }_{-\infty}^{R L} \mathrm{D}_{x_{j}}^{\alpha} B_{x_{i}}^{(1)}$, and the matrix

Instruments, Ministry of Education of China, Jilin University, Changchun, 130026, China and the College of Instrumentation and Electrical Engineering, Jilin University, Changchun, 130026, China (e-mail: suiyangyi@jlu.edu.cn). 
form is

$$
\boldsymbol{G}^{(1+\alpha)}=\left[B_{x_{i} x_{j}}^{(1+\alpha)}\right]
$$

The scalar potential of the magnetic dipole is [7]

$$
\varphi^{(0)}=-\frac{\mu_{0}}{4 \pi} \boldsymbol{m} \cdot \nabla \frac{1}{r}
$$

Build a Cartesian coordinate system and set the position of the magnetic dipole at its origin. The vector $\boldsymbol{m}\left(m_{x}, m_{y}, m_{z}\right)$ represents the magnetic dipole moment. The vector $\boldsymbol{r}(x, y, z)$ is from the position of the magnetic dipole to the observation point, and its magnitude is $r=\sqrt{x^{2}+y^{2}+z^{2}}$.

Now we take the derivative of $\varphi^{(0)}$ with respect to $z$

$$
\begin{aligned}
{ }_{-\infty}^{R L} \mathrm{D}_{z}^{\alpha} \varphi^{(0)} & ={ }_{-\infty}^{R L} \mathrm{D}_{z}^{\alpha}\left(-\frac{\mu_{0}}{4 \pi} \boldsymbol{m} \cdot \nabla \frac{1}{r}\right) \\
& =-\frac{\mu_{0}}{4 \pi}{ }_{-\infty}^{R L} \mathrm{D}_{z}^{\alpha}\left(m_{x} \frac{\partial}{\partial x} \frac{1}{r}+m_{y} \frac{\partial}{\partial y} \frac{1}{r}+m_{z} \frac{\partial}{\partial z} \frac{1}{r}\right)
\end{aligned}
$$

${ }_{-\infty}^{R L} \mathrm{D}_{z}^{\alpha} \varphi^{(0)}$ represents taking the $\alpha$ th-order fractional derivative of $\varphi^{(0)}$ with respect to $z$, where $\alpha>0$ and $\alpha \in \mathbb{R}$, according to the Riemann-Liouville definition of fractional derivatives. Since the operator $\frac{\partial}{\partial x}$ and $\frac{\partial}{\partial y}$ are derivatives with respect to $x$ and $y$ while ${ }_{-\infty}^{R L} \mathrm{D}_{z}^{\alpha}$ is that of $z$, the fractional derivatives and the integer derivatives of the first two terms in (4) are commutative. According to the composition rules [8] between the integer-order and fractional-order derivatives, we have

$$
\begin{aligned}
& { }_{-\infty}^{R L} \mathrm{D}_{t}^{\alpha}\left(\frac{\mathrm{d}^{n}}{\mathrm{~d} t^{n}} f(t)\right) \\
= & \frac{\mathrm{d}^{n}}{\mathrm{~d} t^{n}}\left({ }_{-\infty}^{R L} \mathrm{D}_{t}^{\alpha} f(t)\right)-\lim _{t_{0} \rightarrow-\infty} \sum_{k=0}^{n-1} \frac{f^{(k)}\left(t_{0}\right)\left(t-t_{0}\right)^{k-\alpha-n}}{\Gamma(1+k-\alpha-n)}
\end{aligned}
$$

where $n \in \mathbb{N}, \alpha \in \mathbb{R} ; \Gamma(\cdot)$ refers to gamma function. Equation (5) shows that there is a summation remainder after exchanging the fractional-order derivatives and the integer-order derivatives. Due to $\lim _{r \rightarrow-\infty} \frac{1}{r}=0$, the remainder of the third term is equal to 0 . In fact, not only can we exchange the fractionalorder and the integer-order derivatives with respect to $z$ without considering the remainder, but also with respect to the other two variables $x$ and $y$. We introduce a new symbol $\varphi_{x_{j}}^{(\alpha)}$ to represent ${ }_{-\infty}^{R L} \mathrm{D}_{X_{j}}^{\alpha} \varphi^{(0)}$, and then (4) can be generalized as

$$
\varphi_{x_{j}}^{(\alpha)}={ }_{-\infty}^{R L} \mathrm{D}_{x_{j}}^{\alpha} \varphi^{(0)}=-\frac{\mu_{0}}{4 \pi} \boldsymbol{m} \cdot \nabla\left({ }_{-\infty}^{R L} \mathrm{D}_{x_{j}}^{\alpha} \frac{1}{r}\right)
$$

We cite one of Engheta's conclusions [3],

$$
{ }_{-\infty}^{R L} \mathrm{D}_{x_{j}}^{\alpha} \frac{1}{r}=\frac{\Gamma(1+\alpha)}{r^{1+\alpha}} \mathrm{P}_{\alpha}\left(-\frac{x_{j}}{r}\right)
$$

where $\mathrm{P}_{\alpha}(\cdot),(\alpha \in \mathbb{C})$ refers to the generalized Legendre function, which can also be expressed by Gauss hypergeometric function [9] as

$$
\mathrm{P}_{\alpha}(t)={ }_{2} \mathrm{~F}_{1}\left(-\alpha, \alpha+1 ; 1 ; \frac{1-t}{2}\right),(\alpha, t \in \mathbb{C})
$$

Substitute (7) into (6), then we obtain the expression of the fractional derivatives of the magnetic dipole potential with respect $x_{j}$

$$
\varphi_{x_{j}}^{(\alpha)}=\frac{-\mu_{0} \Gamma(1+\alpha)}{4 \pi} \boldsymbol{m} \cdot \nabla\left(\frac{\mathrm{P}_{\alpha}\left(-\frac{x_{j}}{r}\right)}{r^{1+\alpha}}\right)
$$

The relationship between the magnetic scalar potential and the magnetic field is [7]

$$
-\nabla \varphi^{(0)}=\boldsymbol{B}^{(1)}
$$

Take the $\alpha$ th-order derivative of both sides of (10), $\alpha \in[0,1]$

$$
{ }_{-\infty}^{R L} \mathrm{D}_{x_{j}}^{\alpha} \nabla \varphi^{(0)}={ }_{-\infty}^{R L} \mathrm{D}_{x_{j}}^{\alpha} \boldsymbol{B}^{(1)}
$$

Exchange the fractional derivative and the gradient operator on the left side of (11)

$$
\begin{aligned}
-\nabla\left({ }_{-\infty}^{R L} \mathrm{D}_{x_{j}}^{\alpha} \varphi^{(0)}\right) & ={ }_{-\infty}^{R L} \mathrm{D}_{x_{j}}^{\alpha} \boldsymbol{B}^{(1)} \\
-\nabla \varphi_{x_{j}}^{(\alpha)} & =\boldsymbol{B}_{x_{j}}^{(1+\alpha)}
\end{aligned}
$$

Expand the operator $\nabla$ and rewrite $\boldsymbol{B}$ with its components

$$
-\sum_{i}\left(\frac{\partial}{\partial x_{i}} \boldsymbol{e}_{\boldsymbol{i}}\right) \varphi_{x_{j}}^{(\alpha)}=\sum_{i} B_{x_{i} x_{j}}^{(1+\alpha)} \boldsymbol{e}_{\boldsymbol{i}}
$$

where $\boldsymbol{e}_{\boldsymbol{i}}(i=1,2,3)$ are basis vectors align the three axes.

After substituting the expression of $\varphi_{x_{j}}^{(\alpha)}$ into (13), we have

$$
B_{x_{i} x_{j}}^{(1+\alpha)}=\frac{\mu_{0} \Gamma(\alpha+1)}{4 \pi} \frac{\partial}{\partial x_{i}}\left(\boldsymbol{m} \cdot \nabla \frac{\mathrm{P}_{\alpha}\left(-\frac{x_{j}}{r}\right)}{r^{1+\alpha}}\right)
$$

Expand (14) and then we obtain the analytic expression of each component of the fractional gradient tensor of the magnetic dipole

$$
\begin{aligned}
& B_{x_{i} x_{j}}^{(1+\alpha)}= \begin{cases}k_{1}\left[a \mathrm{P}_{1+\alpha}(t)+b \mathrm{P}_{2+\alpha}(t)\right] & i=j \\
k_{2}\left[c \mathrm{P}_{\alpha}(t)+d \mathrm{P}_{1+\alpha}(t)+e \mathrm{P}_{2+\alpha}(t)\right] & i \neq j\end{cases} \\
& \text { where }
\end{aligned}
$$

$$
\begin{aligned}
t & =\frac{-x_{j}}{r} \\
k_{1} & =\frac{\mu_{0}(1+\alpha)(2+\alpha) \Gamma(1+\alpha)}{4 \pi\left(x_{j}^{2}-r^{2}\right) r^{3+\alpha}} \\
k_{2} & =\frac{\mu_{0}(1+\alpha) \Gamma(1+\alpha)}{4 \pi\left(x_{j}^{2}-r^{2}\right)^{2} r^{3+\alpha}} \\
a & =r \boldsymbol{m} \cdot \boldsymbol{r}-m_{i} x_{i} r \\
b & =x_{i} \boldsymbol{m} \cdot \boldsymbol{r}-m_{i} r^{2} \\
c & =m_{i} r^{2}\left(r^{2}-x_{j}^{2}\right)-x_{i} r^{2}\left(\boldsymbol{m} \cdot \boldsymbol{r}-m_{j} x_{j}\right)(3+\alpha) \\
d & =m_{j} x_{i} r\left[r^{2}(2+\alpha)+x_{j}^{2}(3+\alpha)\right] \\
& -x_{j} r\left[m_{i}\left(x_{j}^{2}-r^{2}\right)+x_{i} \boldsymbol{m} \cdot \boldsymbol{r}(5+2 \alpha)\right] \\
e & =x_{i} x_{j}\left(m_{j} r^{2}-\boldsymbol{m} \cdot \boldsymbol{r} x_{j}\right)(2+\alpha)
\end{aligned}
$$

\section{COMPARISON BetweEn ANALYTICAL AND NumERICAL} METHOD

The fractional derivatives can be regarded as the transition between the derivatives of the neighboring integral order. When the derivative order changes from fraction to integer, the fractional derivatives should smoothly transform to the integral derivatives. From (2) and (14), we know that when $\alpha=0$, the matrix becomes 


$$
\boldsymbol{G}^{(1)}=\frac{\mu_{0}}{4 \pi}\left[\begin{array}{lll}
\frac{\partial\left(\boldsymbol{m} \cdot \nabla \frac{1}{r}\right)}{\partial x_{1}} & \frac{\partial\left(\boldsymbol{m} \cdot \nabla \frac{1}{r}\right)}{\partial x_{1}} & \frac{\partial\left(\boldsymbol{m} \cdot \nabla \frac{1}{r}\right)}{\partial x_{1}} \\
\frac{\partial\left(\boldsymbol{m} \cdot \nabla \frac{1}{r}\right)}{\partial x_{2}} & \frac{\partial\left(\boldsymbol{m} \cdot \nabla \frac{1}{r}\right)}{\partial x_{2}} & \frac{\partial\left(\boldsymbol{m} \cdot \nabla \frac{1}{r}\right)}{\partial x_{2}} \\
\frac{\partial\left(\boldsymbol{m} \cdot \nabla \frac{1}{r}\right)}{\partial x_{3}} & \frac{\partial\left(\boldsymbol{m} \cdot \nabla \frac{1}{r}\right)}{\partial x_{3}} & \frac{\partial\left(\boldsymbol{m} \cdot \nabla \frac{1}{r}\right)}{\partial x_{3}}
\end{array}\right]
$$

In (16), each row is the same, and each column denotes the three components of the magnetic field of the magnetic dipole. When $\alpha=1$, the matrix becomes

$$
\boldsymbol{G}^{(2)}=\frac{-\mu_{0}}{4 \pi}\left[\begin{array}{lll}
\frac{\partial\left(\boldsymbol{m} \cdot \nabla \frac{x_{1}}{r^{3}}\right)}{\partial x_{1}} & \frac{\partial\left(\boldsymbol{m} \cdot \nabla \frac{x_{2}}{r^{3}}\right)}{\partial x_{1}} & \frac{\partial\left(\boldsymbol{m} \cdot \nabla \frac{x_{3}}{r^{3}}\right)}{\partial x_{1}} \\
\frac{\partial\left(\boldsymbol{m} \cdot \nabla \frac{x_{1}}{r^{3}}\right)}{\partial x_{2}} & \frac{\partial\left(\boldsymbol{m} \cdot \nabla \frac{x_{2}}{r^{3}}\right)}{\partial x_{2}} & \frac{\partial\left(\boldsymbol{m} \cdot \nabla \frac{x_{3}}{r^{3}}\right)}{\partial x_{2}} \\
\frac{\partial\left(\boldsymbol{m} \cdot \nabla \frac{x_{1}}{r^{3}}\right)}{\partial x_{3}} & \frac{\partial\left(\boldsymbol{m} \cdot \nabla \frac{x_{2}}{r^{3}}\right)}{\partial x_{3}} & \frac{\partial\left(\boldsymbol{m} \cdot \nabla \frac{x_{3}}{r^{3}}\right)}{\partial x_{3}}
\end{array}\right]
$$

Equation (17) is the same as the expression of the magnetic gradient tensor of the magnetic dipole. When $\alpha \in(0,1)$, the expressions can be verified by comparing with the numerical method. Because the Riemann-Liouville and the GrunwaldLetnikov derivatives are the same when applying them to calculating $\frac{1}{r}$ [8], we use Xue's glfdiff2() numerical function which implements the high-precision numerical algorithm of Grunwald-Letnikov fractional derivative [10]. We compare the results of glfdiff2() with those of the analytic expression (15). The simulations are as follows:

Set the position of the magnetic dipole at the origin of a coordinate system. The dipole's moment is $\left(m_{1}, m_{2}, m_{3}\right)=(0,0$, $80)$. The equation of the survey line is

$$
\left\{\begin{array}{l}
x=t \\
y=0 \\
z=100
\end{array}, t \in(-500,500)\right.
$$

The $B_{x x}^{(1+\alpha)}$ on the survey line are calculated by the numerical algorithm and the analytic expression respectively with $\alpha=0.2,0.4,0.6$, and 0.8 , and the results are shown in Fig. 1. The solid lines and diamond lines are the value calculated by the numerical and analytical method respectively. The solid and diamond lines are consistent, which shows the correctness of the proposed analytical expression.
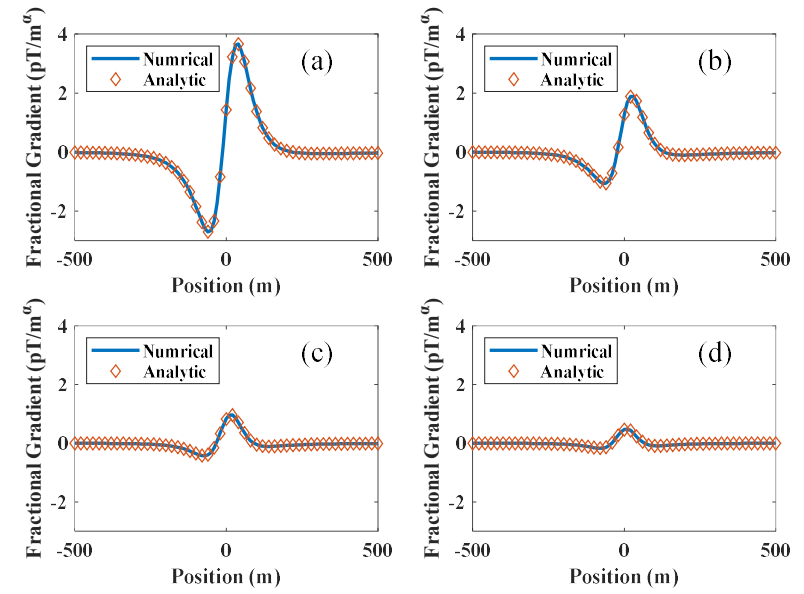

Fig. 1. (a) (b) (c) (d) are the comparisons of the numerical and analytic results with $\alpha=0.2,0.4,0.6$, and 0.8 . The horizontal axis represents the positions of the observation points on the survey line, and the vertical axis represents the value of the fractional derivatives whose unit are $\mathrm{T} / \mathrm{m}^{\alpha}$.

\section{POTENTIAL APPLICATIONS}

\section{A. Expansion of sensing area of magnetic gradient tensor}

Fig. 1 shows that as the derivative order $\alpha$ increases, the value of the fractional derivatives on the survey line decrease gradually. If $\alpha$ is fixed on zero or one, the current level of measurement, the attenuation property of potential fields causes a big blind sensing area in practical survey due to the limited resolution of magnetic sensors. The following simulations show the maximum sensing area of the gradiometer with different derivative orders.

Since the unit of fractional magnetic gradient tensor is $\mathrm{T} / \mathrm{m}^{\alpha}$, the fractional tensor components are multiplied by $\mathrm{m}^{\alpha-1}$ to normalize the unit to $\mathrm{T} / \mathrm{m}$. Let the resolution of a magnetic gradient sensor $\operatorname{Res}_{\min }$ be $1 \mathrm{pT} / \mathrm{m}$ and the properties of the magnetic dipole are same as those in the above-mentioned simulations. The survey plane is the $x-y$ plane with $z=100 \mathrm{~m}$, and the size of the survey area is $1000 \mathrm{~m} \times 1000 \mathrm{~m}$. Calculate the maximum available sensing area of the different fractional magnetic gradient tensor of the magnetic dipole with $\alpha=0,0.2$, $0.4,0.6,0.8$, and 1 by $\left|B_{x x_{i}}^{(1+\alpha)}\right|=\operatorname{Res}_{\text {min }}$. The result is shown in Fig. 2 and the available sensing areas are encircled by several closed colored curves. The area enclosed by the red line, the available sensing range of the magnetic field, is the largest, and the area enclosed by the black line, the available sensing range of the magnetic gradient tensor, is the smallest. There is a big blind area between them. The ranges of the sensing areas of the fractional magnetic gradient tensor are intermediate and fill the gap between them.

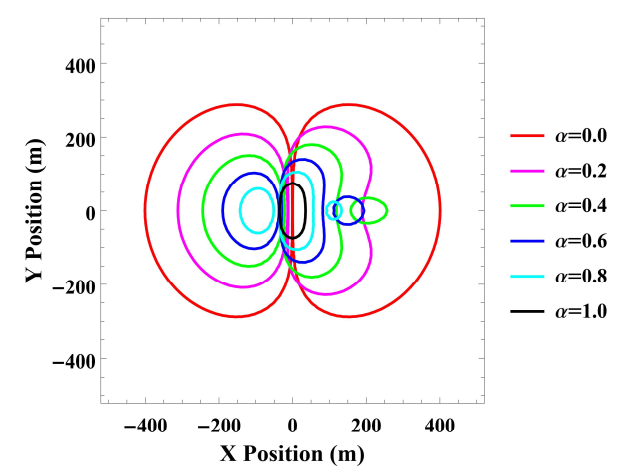

Fig. 2. The colored curves indicate the maximum available sensing areas of the gradient tensors with various derivative orders.

\section{B. Ambiguity elimination in magnetic dipole localization}

For the location problem of a magnetic dipole, it is insufficient to obtain its accurate position using the magnetic gradient tensor only [5]. The key reason is that only five of the nine components are independent and six parameters are needed to be solved. However, (14) shows that the fractional magnetic gradient tensor $\boldsymbol{G}^{(1+\alpha)}$ is asymmetric, which increases the numbers of independent equations for solving the localization of the magnetic dipole. 
To demonstrate this feature of the fractional magnetic gradient tensor of the magnetic dipole, we calculate the tensors on the $x-y$ observation plane with $z=0.5$ and $\alpha$ from 0 to 1 , and stack the tensors along $\mathrm{z}$ axis. The results are displayed by an open softeware VIS5D [11] and shown in Fig. 3.

The nine components of the fractional magnetic gradient tensor are displayed accroding to the position of the magnetic gradient tensor matrix. The bottom of the stereogram means $\alpha$ is zero and $\boldsymbol{G}^{(1)}$ is composed of three magnetic field components The top of the stereogram means $\alpha$ is one and $\boldsymbol{G}^{(2)}$ becomes the magnetic gradient tensor which are symmetric in the matrix. However, if $\alpha \in(0,1)$, the parts between the bottom and top show that the tensors on the $x-y$ observation plane with the same $\alpha$ are asymmetric and gradually become symmetric due to the transitional property of fractional derivatives.
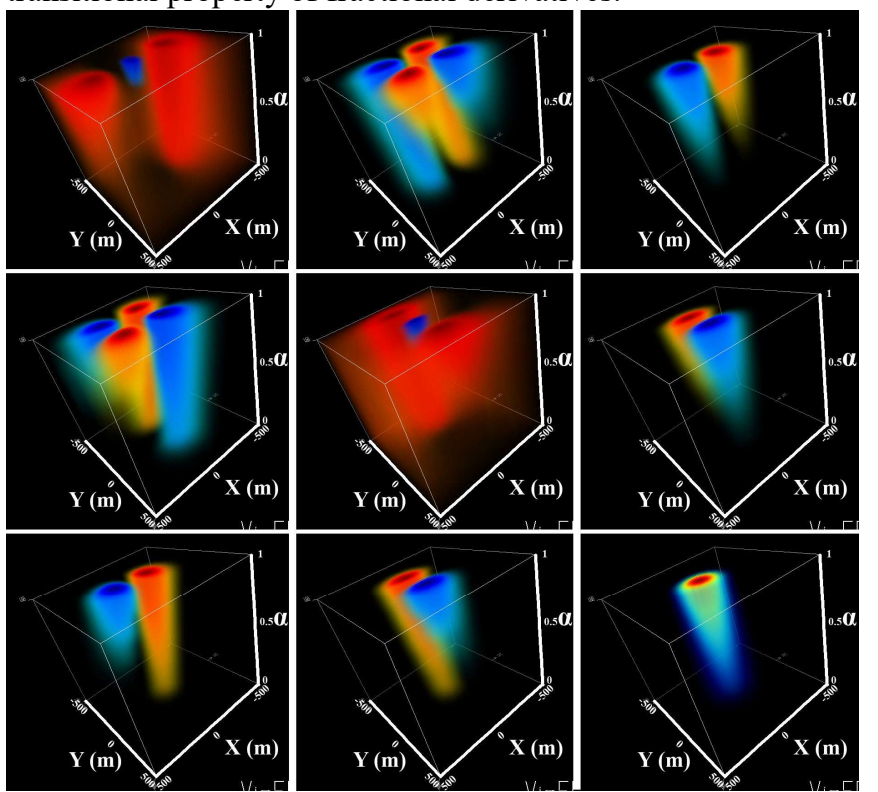

Fig. 3. These nine graphs show the nine components of the fractional magnetic gradient tensor respectively. The $x$ and $y$ axes in each graph correspond to that of the observation plane, and the $z$ axis represents the derivative order $\alpha$. Note: VIS5D cannot customize the labels of the coordinate axis, the white coordinate axes and labels are added by a painting tool for clarity.

\section{CONCLUSION}

In this paper, we deduce the analytic expression of the fractional magnetic gradient tensor of the magnetic dipole using the sequential derivative between the fractional-order and the integer-order derivatives, and then verify the analytic expression by comparing the analytical results with the numerical results. Finally, we discuss its potential applications. One is expanding the available sensing range of the magnetic gradient tensor by changing the value of the derivative order. The other is solving the multiple solution problems of magnetic dipole localization by the nine independent components of the fractional gradient tensor. Fractional gradient tensors have many rich and interesting properties being worth digging further.

\section{ACKNOWLEDGMENT}

The word modification suggestion was made by Xiaomeng Lei.

\section{REFERENCES}

[1] R. Hilfer, "An Introduction to Fractional Calulus," in Applications of Fractional Calculus in Physics, Singapore, World Scientific Publishing, 2000, ch. 1, sec. 1, pp. 3-6.

[2] G. Cooper and D. Cowan, "The Application of Fractional Calculus to Potential Field Data," Exploration Geophysics, vol. 34, no. 1-2, pp. 51-56, Jan. 2003, 10.1071/EG03051.

[3] N. Engheta, "On fractional calculus and fractional multipoles in electromagnetism," IEEE Transactions on Antennas and Propagation, vol. 44, No. 4, pp. 554-566, Apr. 1996, 10.1109/8.489308.

[4] Y. Sui, K. Leslie and D. Clark, "Multiple-Order Magnetic Gradient Tensors for Localization of a Magnetic Dipole," IEEE Magnetics Letters, vol. 8, pp. 1-5, Jun. 2017, 10.1109/LMAG.2017.2708682.

[5] D. A. Clark, "Corrigendum to: New Methods for Interpretation of Magnetic Vector and Gradient Tensor Data I: Eigenvector Analysis and the Normalized Source Strength," Exploration geophysics (Melbourne), vol. 43, no. 4, pp. 267-782, Sep. 2014, 10.1071/EG12020_CO.

[6] L. B. Pedersen and T. M. Rasmussen, "The Gradient Tensor of Potential Field Anomalies: Some Implications on Data Collection and Data Processing of Maps," Geophysics, vol. 55, no. 12, pp. 1558-1566, Dec. 1990, 10.1190/1.1442807.

[7] R. J. Blakely, "Magnetic Potential," Potential Theory in Gravity and Magnetic Applications, NewYork, NY, USA: Cambridge University Press, 1996, pp. 70-73.

[8] I. Podlubny, "Fractional Derivatives and Integrals," Fractional Differential Equations, San Diago, CA, USA: Academic Press, 1999, ch. 2, sec. 3, pp. 74-77.

[9] Z. Wang and D. Guo, "Legendre Function," Introduction to Special Function, Beijin, China, Peking Univ., 2000, ch.5, sec. 16 , pp. 241-243.

[10] D. Xue, "The Definition and Calculation of Fractional Calculus," Fractional Calculus and Fractional-order Control, Beijin, China, China Science Publishing, 2018, ch. 3 , sec. 4 , pp. 55-56.

[11] W. L. Hibbard, et al., "Interactive visualization of Earth and space science computations," Computer, vol. 27, no. 7, pp. 65-72, Jul. 1994, 10.1109/2.299413. 